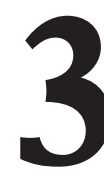

\title{
CIBERACOSO EN ESTUDIANTES UNIVERSITARIOS ANTES Y DURANTE EL CONFINAMIENTO POR LA COVID-19
}

\section{(CYBERBULLYING IN UNIVERSITY STUDENTS BEFORE AND AFTER COVID-19 LOCKDOWN)}

María Jesús Caurcel Cara

Emilio Crisol Moya

Universidad de Granada

DOI: $10.5944 / e d u c X X 1.30525$

Cómo referenciar este artículo/How to reference this article:

Caurcel Cara, M.J. y Crisol Moya, E. (2022). Ciberacoso en estudiantes universitarios antes y durante el confinamiento por la COVID-19. Educación XX1, 25(1), 67-91. https://doi.org/10.5944/ educXX1.30525

Caurcel Cara, M.J., \& Crisol Moya, E, (2022). Cyberbullying in university students before and after COVID-19 lockdown. Educación XX1, 25(1), 67-91. https://doi.org/10.5944/educXX1.30525

\section{RESUMEN}

El ciberacoso en el contexto universitario constituye una realidad menos estudiada en comparación con su investigación en etapas educativas no universitarias. El objetivo del estudio es analizar el ciberacoso en estudiantes universitarios antes y durante el confinamiento por la Covid-19 estableciendo los roles del ciberacoso, los afectos experimentados, las relaciones con los iguales y el uso social de Internet. Se utilizó un diseño transversal no experimental, ambispectivo, descriptivo e inferencial, valiéndose de tres cuestionarios estandarizados y validados, y uno ad hoc para evaluar la influencia del confinamiento. Participaron 586 estudiantes $\left(M_{\mathrm{Edad}}=22.67\right.$; $D T=5.75 ; 81.1 \%$ mujeres) de Grado y Máster de la Universidad de Granada (España), principalmente de las ramas de Ciencias Sociales y Jurídicas (44.20\%) y de Ciencias de la Salud (28.31\%). Antes del confinamiento, el 
$16.2 \%$ de los participantes sufrieron cibervictimización, el 6.7\% acosaron a otros, el $8.4 \%$ fueron víctimas/agresores y el $68.8 \%$ sin rol. Durante el confinamiento, los porcentajes de víctimas y víctimas/agresores descendieron, aumentado los agresores. Se encontró una influencia del sexo, la edad y los afectos positivos en los roles antes del confinamiento $(p<.05)$, y de la edad, los afectos negativos y las relaciones con los iguales durante el confinamiento $(p<.05)$. Las víctimas fueron las que más afectos negativos experimentaron y las que más apoyo recibieron de los compañeros durante el confinamiento. El descenso de la victimización durante el confinamiento puede tener relación con el mayor apoyo recibido por las victimas que ha actuado como factor de protección. Los resultados reflejan que aún se debe incidir en un uso positivo, no nocivo y responsable de los teléfonos móviles entre los jóvenes. Desde la universidad se debe favorecer un clima institucional positivo e integrador que ayude a intervenir, pero sobre todo para prevenir el ciberacoso.

\section{PALABRAS CLAVE}

Ciberacoso, estudiantes universitarios, afectividad, relaciones sociales, Covid-19

\section{ABSTRACT}

Cyberbullying in the university context is a little-studied reality. The aim of this study is to analyse cyberbullying in university students before and after the lockdown due to Covid-19, establishing the roles of cyberbullying, the affects experienced, peer relations and social use of the internet. We have used a non-experimental, ambispective, descriptive and inferential cross-sectional design, employing three standardized and validated questionnaires, and one ad-hoc, in order to assess the influence of the lockdown. 586 students $\left(M_{\text {Age }}=\right.$ 22.67; $S D=5.75 ; 81.1 \%$ female) of Degrees and Masters at the University of Granada (Spain) participated, mainly from Social and Legal Sciences (44.20\%) and Health Sciences (28.31\%). Before the lockdown, $16.2 \%$ of the participants were cyber-victimized, $6.7 \%$ had bullied, $8.4 \%$ were victims/aggressors and $68.8 \%$ had no role. During the lockdown, the percentages of victims and victims/ aggressors decreased, while the percentage of aggressors rose. We found there to be an influence of sex, age and positive affects in the roles before lockdown $(p<.05)$, and of age, negative affects and peer relations during the lockdown $(p<.05)$. The victims were those who experienced the most negative effects and those who received most support from their fellow students during the lockdown. The decrease in victimization during the lockdown may be related to the greater support received by the victims, which acted as a protecting factor. The results show that emphasis should still be placed on a positive, 
non-harmful and responsible use of mobile telephones among young people. The university should promote a positive and inclusive institutional climate that helps to intervene, but above all to prevent cyberbullying.

\section{KEYWORDS}

Cyberbullying, college students, affect, interpersonal relationships, Covid-19

\section{INTRODUCCIÓN}

El teléfono móvil se ha convertido en la tecnología más popular entre los adolescentes y jóvenes, y en una herramienta imprescindible en su vida diaria (Abu Bakar, 2015; Polo et al., 2017). El uso cada vez mayor del teléfono móvil y la sobreexposición a las redes sociales ha generado una cultura del ciberespacio (Dennehy et al., 2020; Rodríguez-Correa y Rivaudalla, 2018) conllevando importantes cambios en las formas de comunicación y relación, así como en los espacios y contextos en los que esa interacción se da (Baldry et al., 2015; Dobarro et al., 2018; Redondo et al., 2017); llegando a ser el único medio para mantener el contacto social y/o establecer relaciones sociales, durante el confinamiento impuesto por la pandemia de la Covid19 (Mkhize y Gopal, 2021).

En España para detener la propagación del virus se aplicaron medidas de distanciamiento social, llevando al confinamiento de la población no esencial el 14 de marzo de 2020. La excepcionalidad derivada del mismo ha afectado a todos los ámbitos de nuestro funcionamiento como sociedad: sanitario, económico, educativo, y por supuesto el relacional (Lozano-Díaz et al., 2020). Como consecuencia, la gente ha recurrido a Internet y a los medios sociales para sus actividades diarias, participando y pasando más tiempo en línea. Aumentando especialmente el uso del teléfono móvil hasta en $38.3 \%$ ( 3 horas), con fines de comunicación, entretenimiento, teletrabajo y/o educativos (Smartme Analytics, 2020).

Antes del estallido de la Covid-19 y el cierre de la universidad, el teléfono móvil ha estado muy presente en los campus (Polo et al., 2017; Watts et al., 2017). Los estudiantes lo han utilizado tanto con fines educativos, para buscar información, comunicarse, organizarse y colaborar con sus compañeros (Tindell y Bohlander, 2012), como lúdicos, en su tiempo de ocio (Barkley y Lepp, 2013). Sin embargo, también se ha utilizado para acosar, hostigar, excluir e intimidar a los otros (Gámez-Guadix et al., 2013; Myers y Cowie, 2013; Ortega-Barón et al., 2016; Wegge et al., 2016; Zalaquett y Chatters, 2014). 
A pesar de que el ciberacoso en el contexto universitario constituye una realidad (Khine et al., 2020; Martínez-Ramón et al., 2020; Watts et al., 2017), ha carecido del protagonismo otorgado en etapas educativas previas (Dobarro et al., 2018; Khine et al., 2020; Martínez-Monteagudo et al., 2020; Martínez-Ramón et al., 2019; Rodríguez-Correa y Rivaudalla, 2018).

De hecho, como indican Bernardo et al. (2020) se ha venido cuestionando su existencia, pues se le atribuía cierta madurez psicosocial a la población universitaria, que hacía pensar que los problemas en las relaciones con los iguales se minimizarían (Méndez et al., 2019). Nada más lejos de la realidad, la etapa universitaria es un periodo de transición que en muchos casos conlleva la independencia del núcleo familiar, estrés ante la nueva situación o búsqueda de nuevas amistades, cambios en los usos de Internet -ahora sin supervisión parental-, etc., que ponen en riesgo de sufrir o ejercer comportamientos relacionados con el ciberacoso (Dobarro et al., 2018; Khine et al., 2020; Martínez-Monteagudo et al., 2020; Polo et al., 2017), sobre todo en las minorías étnicas-culturales y/o sexuales, y en estudiantes con capacidades académicas diferentes o con necesidades especiales (Méndez et al., 2019; Wensley y Campbell, 2012).

En los últimos tiempos se ha constado un incremento del ciberacoso entre universitarios (Bernardo et al., 2020; Dobarro et al., 2017; MartínezMonteagudo et al., 2020), con tasas de prevalencia muy variables (Faucher et al., 2014), entre el 10\% y el 60\% en diferentes países (Khine et al., 2020). En universidades españolas oscila entre el 20\% y el 50\% (Vergel et al., 2016). Entre los roles implicados, víctima, agresor, espectador y victima/agresor (Martínez-Ramón et al., 2019), se han encontrado diferentes tasas de prevalencia en función de las metodologías utilizadas y la población objetivo de estudio. En estudios con universitarios españoles, el 9.16\% es identificado como víctima, el $15.3 \%$ como agresor, el $18.1 \%$ como víctima/agresor y el $50.4 \%$ sin rol (Polo et al., 2017). Dobarro et al. (2018) han encontrado que el $22.1 \%$ confiesa ser víctima de acoso, $1.1 \%$ de forma severa, y un $29.3 \%$ de los estudiantes han observado conductas de acoso. Por su parte, MartínezMonteagudo et al. (2019) han señalado que el 18.6\% han sido cibervíctimas y el 19.4\% ciberagresores. En el estudio de Méndez et al. (2019) el 14.4\% de los estudiantes ha sido objeto de ciberacoso, el 7.3\% ha realizado ciberacoso y el $17.4 \%$ lo ha observado.

Tanto hombres como mujeres aparecen implicados en el ciberacoso, pero existe una falta de consistencia respecto a la variable sexo en los resultados obtenidos en los estudios previos con universitarios (Dobarro et al., 2017; Faucher et al., 2014). Algunos estudios previos no han observado diferencias de género (Kowalski et al., 2012). Mientras que en otros, los varones han sido los que están más implicados en el ciberacoso 
(Durán-Segura y Martínez-Pecino, 2015; Navarro et al., 2016), puntuando más alto en conductas agresivas (Sánchez et al., 2011) y las mujeres las que más lo han sufrido (Khine et al., 2020; Zalaquett y Chatters, 2014). En cambio en otros estudios ocurre al contrario, los hombres se han visto más implicados en los roles de víctimas y las mujeres en el de agresoras (Dobarro et al., 2017; Martínez-Ramón et al., 2019, Méndez et al., 2019).

La incidencia de la edad en el ciberacoso aún no está clara. Existen estudios que sugieren que disminuye con la edad (Garaigordobil y Oñederra, 2009; Ševčíková y Šmahel, 2009), por lo que debería disminuir en la transición de la Educación Secundaria a la Educación Superior. En cambio, otros autores (Faucher et al., 2014; Kiriakidis y Kavoura, 2010; Kowalski et al., 2012; Washington, 2015) han afirmado lo contrario, que aumenta en la secundaria y que continúa en la etapa universitaria.

En el ciberacoso los diferentes roles implicados experimentan un amplio espectro de emocionalidad y consecuencias psicológicas de estos impactos afectivos (Vallés, 2014). Ortega-Ruiz et al. (2012) afirman que numerosos estudios asocian la cibervictimización con sentimientos negativos. En este sentido, las víctimas se sienten inseguras, angustiadas, asustadas y avergonzadas, experimentando estrés, ansiedad o depresión (Finkelhor et al., 2000; Khine et al., 2020). Beran y Li (2005) han señalado que las respuestas emocionales de las víctimas han sido enojo, tristeza, dolor, ansiedad, vergüenza, miedo y autoculpa. En la misma línea, McLoughlin et al. (2013) afirman que han sentido enojo, molestia, vergüenza, inseguridad, disgusto y decepción, así como consecuencias sociales de exclusión y emocionales, como la falta de afrontamiento efectivo o la autoinculpación. En cambio, en los estudios de Hoff y Mitchell (2009) y Patchin y Hinduja (2012) han experimentado ira, impotencia, frustración, depresión y temor, que pueden dar lugar a trastornos psicológicos, y aquellas víctimas que sienten ira pueden cambiar de rol comenzando a acosar a otros. Las víctimas/agresores han experimentado frialdad emocional o indiferencia afectiva (Ortega-Ruiz et al., 2009). En el agresor se ha producido un efecto desinhibidor (Mason, 2005), favorecido por la sensación de distancia y de supuesto anonimato del ciberespacio (Avilés, 2013). El agresor debido a su falta de empatía no ha experimentado sentimientos de culpa, compasión, arrepentimiento o malestar emocional (Martínez-Monteagudo et al., 2020), sino una sensación placentera ante las agresiones, pudiéndose hablar de la desconexión moral del agresor (Avilés, 2013). En los espectadores se ha producido una emocionalidad ambivalente en función de la tipología defensores, reforzadores, compinches o ajenos (Vallés, 2014).

Los afectos experimentados por los diferentes roles y las situaciones de ciberacoso pueden verse intensificados por la falta de apoyo social de los 
iguales (Calvete et al., 2010; Ortega-Barón et al., 2016; Navarro et al., 2015). En los estudios de Calvete et al. (2010) y Katzer et al. (2009) los agresores se han caracterizan por su bajo apoyo social. En cambio, Romera et al. (2016) los caracterizan con un mayor apoyo social y a las víctimas con el menor apoyo.

Wright (2016) ha descubierto que las cibervíctimas utilizaban estrategias de afrontamiento de apoyo social (adaptativas) y de venganza (desadaptativas), o una combinación de ambas, para hacer frente al acoso. Worsley et al. (2019) han encontrado que el apoyo de los compañeros confiere resiliencia contra el ciberacoso. El sentimiento de pertenencia al grupo, la reciprocidad, la competencia social, la aceptación por los iguales se vinculan con el bienestar psicológico, social y emocional (Romera et al., 2016). Esta importancia del apoyo social de los iguales se ha vuelto, si cabe, aún más determinante en el periodo de confinamiento vivido por la Covid19. Forzosamente la interacción social con los iguales tiene que realizarse en el ciberespacio, y su apoyo es básico no solo para protegerse del ciberacoso, sino también para afrontar el aislamiento saludablemente.

Aunque aún son pocos los estudios que han abordado el ciberacoso durante el confinamiento, los mismos apuntan a que un mayor tiempo navegando puede hacer que el número de casos se haya acentuado (GómezLeón, 2021; Mkhize y Gopal, 2021; Yahya et al., 2020). En un estudio con adolescentes, Gómez-León (2021) ha encontrado un descenso en el ciberacoso en el caso de las víctimas durante el confinamiento. Relacionando este descenso tanto con el mayor control parental ejercido durante la pandemia y con haber compartido más tiempo con sus cuidadores, acompañado de mayor apoyo familiar y social. Con estudiantes universitarios, Alsawalqa (2021) ha encontrado que los estudiantes varones de ascendencia asiática oriental y sudoriental han sufrido más ciberacoso en forma de discriminación racial durante el confinamiento.

Teniendo en cuenta todo lo anterior, el estudio tuvo como objetivo general analizar el ciberacoso en estudiantes universitarios antes y durante el confinamiento por la Covid-19. Y como objetivos específicos: a) establecer los roles y perfiles en el ciberacoso antes y durante el confinamiento; b) determinar los afectos experimentados por los diferentes roles antes y durante el confinamiento; c) analizar por roles las relaciones sociales con los iguales antes y durante el confinamiento y la docencia online; y, d) identificar el uso social que hacen de Internet los diferentes roles durante el confinamiento.

Así, a modo de juicio empírico, se plantean las siguientes hipótesis: 
- H1: El ciberacoso en estudiantes universitarios ha aumentado durante el confinamiento.

- H2: Los perfiles han sido diferentes antes y durante el confinamiento.

- H3: En los perfiles ha influido la edad, el sexo, los afectos y la valoración de las relaciones con los iguales.

- H4: Las víctimas han experimentado más afectos negativos que el resto de roles antes y durante el confinamiento.

- H5: Las víctimas han valorado de forma más negativa las relaciones con los iguales, antes y durante el confinamiento, y la docencia online.

- H6: Las víctimas han valorado de forma más negativa las relaciones con los iguales durante la docencia online.

- H7: No ha habido diferencias por roles en el uso de Internet durante el confinamiento.

\section{MATERIALES Y MÉTODO}

El estudio realizado respondió a un diseño de investigación empírica, de tipo transversal, ambispectivo (retrospectivo, antes del confinamiento y prospectivo, durante), no experimental, descriptiva e inferencial, desarrollado bajo los presupuestos del paradigma metodológico cuantitativo (Hernández et al., 2010).

\section{Participantes}

Para la selección de los participantes se utilizó un muestreo no probabilístico por bola de nieve, conformando una muestra total de 586 estudiantes de la Universidad de Granada (España). La mayoría eran estudiantes de Grado (86.0\%, $n=504)$ de las ramas de Ciencias Sociales y Jurídicas $(44.20 \%, n=259)$ o de las Ciencias de la Salud $(28.31 \%, n=$ $166)$. El 36.9\% $(n=216)$ cursaba primero, el 18.9\% $(n=111)$ segundo, el $14.5 \%(n=85)$ tercero, el $15.7 \%(n=92)$ cuarto y $14.0 \%(n=82)$ Máster. El rango de edad variaba entre los 18 y 64 años $(M=22.67 ; D T=5.75)$, siendo el $81.1 \%$ mujeres $(n=475)$ y el $18.8 \%(n=110)$ hombres. El $82.1 \%(n=$ 477) eran heterosexuales, el $13.6 \%(n=79)$ bisexuales y el $4.3 \%(n=25)$ homosexuales. Aunque la mayoría no trabajaban $(81.2 \% ; n=476)$, se encontró un $11.9 \%$ que lo hacía antes del confinamiento $(n=70)$ y un $6.8 \%$ 
( $n=40)$ continuó durante el confinamiento. El único criterio de exclusión fue que los estudiantes no hubieran estado confinados, por lo que solo un $0.5 \%(n=3)$ de las encuestas fueron descartadas.

\section{Instrumentos}

Cuestionario sociodemográfico ad hoc, que contenía cuestiones relativas a edad, sexo, orientación sexual, titulación, curso, trabajo y tiempo de confinamiento.

"Escala de Victimización a través del Teléfono Móvil" (CYBVIC) (Buelga et al., 2012) compuesta por 10 ítems que evaluaban cibervictimizaciones de hostigamiento, persecución, denigración, violación de la intimidad y exclusión social sufridas a través del teléfono móvil en un contexto determinado (por ejemplo, "Me han insultado o ridiculizado con mensajes o llamadas por el móvil"). Utilizaba una escala tipo de Likert de cuatro opciones de $1=$ Nunca a $4=$ Siempre. El instrumento poseía unas adecuadas propiedades psicométricas, con un coeficiente alfa de Cronbach de .85 para población adolescente (Buelga et al., 2012) y un $=.75$, una fiabilidad compuesta de .79 y una varianza media extractada (VME) de .50 para población universitaria (Polo et al., 2017). Para el estudio la escala se utilizó para preguntar por la cibervictimización a lo largo del curso académico y luego durante el confinamiento, obteniendo un $\alpha=.80$ para los participantes antes del confinamiento y $\alpha=.74$ durante.

"Escala de Agresión a través del Teléfono Móvil" (CYB-AGRES) (Buelga y Pons, 2012) formada por 10 ítems que evaluaban comportamientos que implicaban ciberagresiones de hostigamiento, persecución, denigración, violación de la intimidad y exclusión social realizadas a través del teléfono móvil en un contexto determinado (por ejemplo "He contado mentiras o rumores falsos sobre alguien"). La escala de respuesta era tipo Likert de cuatro opciones de 1 = Nunca a 4 = Siempre. El instrumento poseía unas adecuadas propiedades psicométricas, con un coeficiente alfa de Cronbach de .88 para población adolescente (Buelga y Pons, 2012) y un $\alpha=.82$, una fiabilidad compuesta de .85 y una varianza media extractada de .52 para población universitaria (Polo et al., 2017). Para el estudio la escala se utilizó para preguntar por las ciberagresiones a lo largo del curso académico y durante el confinamiento, obteniendo un $\alpha=.75$ para los participantes antes del confinamiento y $\alpha=.70$ durante.

"Escala de afecto positivo y negativo" (PANAS) (López-Gómez et al., 2015) es uno de los instrumentos más utilizados y recomendados en la literatura para la evaluación de emociones. Está conformada por 20 ítems, 
con una estructura bifactorial: afecto positivo ("Ilusionado", "Fuerte"...) y afecto negativo (“Asustado", "Nervioso"...), cada una con 10 ítems. El participante debía indicar si había sentido cada afecto en una escala Likert de cinco puntos de 1 = Nada o muy ligeramente a $5=$ Mucho, durante el periodo temporal especificado. La puntuación total de cada subescala oscilaba entre 10 y 50 puntos, de tal manera que una mayor puntuación indicaba mayor presencia del afecto concreto. La PANAS ha mostrado excelentes propiedades psicométricas en diferentes estudios con muestras de adultos y estudiantes. El coeficiente alfa de Cronbach para población española fue de .92 para la subescala de Afecto positivo y de .88 para la subescala de Afecto negativo, siendo de $\alpha=.90$ y de $\alpha=.89$, respectivamente, para los participantes de este estudio.

Cuestionario ah doc sobre las relaciones sociales con los compañeros, en el que se les preguntaba cómo las valoraban (positivas o negativas) antes y durante el confinamiento $(\alpha=.91)$, sobre qué usos le dieron a Internet durante el confinamiento relativos a las relaciones sociales (por ejemplo, para contactar con los compañeros, redes sociales, mensajería, etc.) $(\alpha=.76)$ y sobre cómo fueron las relaciones con los iguales durante la docencia online en el confinamiento (por ejemplo, el apoyo de los compañeros, los trabajos en grupo, etc.) $(\alpha=.70)$.

\section{Procedimiento}

Dado que el estudio se realizó durante el estado de alarma declarado el 14 de marzo de 2020 en España, para afrontar la situación de emergencia sanitaria provocada por la Covid-19, la recogida de datos se realizó online mediante la creación de una encuesta a través de la herramienta de "Doc" de Google/Gmail. La distribución de los instrumentos se realizó a través de medios digitales masivos institucionales de la Universidad de Granada (PRADO2 y listas de distribución) e individuales (correo electrónico y WhatsApp). Antes de acceder a la encuesta se presentaba por escrito la finalidad y objetivos del estudio, dejando un email de contacto para cualquier duda; se explicaba la voluntariedad de la participación, garantizando en todo momento su anonimato - eliminando el Internet Protocol (IP) antes de la descarga de datos de Google Form-y confidencialidad, solicitando su consentimiento informado revocable en cualquier momento. Así, se siguieron las directrices éticas de la American Psychological Association, a pesar de ser una investigación que no provocaba daño o perjuicio alguno a los participantes. La investigación contó con el informe favorable del Comité de Ética en Investigación Humana de la Universidad de Granada (España) [2204/CEIH/2021]. En el momento de la recogida de datos, los estudiantes llevaban confinados entre 10 y 16 semanas. 


\section{ANÁLISIS Y RESULTADOS}

\section{Análisis de datos}

En el análisis de los datos se utilizó el programa estadístico SPSS versión 24.0 para Windows, con un margen de error del $5 \%$ y un nivel de confianza del 95\%. Para conocer la distribución de los resultados se calcularon las frecuencias y porcentajes. Para determinar el rol de cada participante en el ciberacoso, siguiendo las indicaciones de Polo et al. (2017), se calcularon los percentiles de las escalas CYB-VI y CYB-AGRES, y se estableció como punto de corte para determinar la mayor implicación en el rol el percentil 75. Al igual que en los estudios previos se encontró que había participantes que se podían incluir en varios roles, por lo que se realizó un análisis discriminante, para predecir a qué grupo era más probable que perteneciera cada participante. Para establecer si había diferencias estadísticamente significativas entre los roles antes y después del confinamiento se realizó la prueba chi-cuadrado de Pearson $\left(\mathrm{c}^{2}\right)$. Para conocer qué variables predictoras (sociodemográficas, académicas, afectivas y sociales) influían en cada rol antes y durante el confinamiento (variable criterio) se calculó un modelo de clasificación mediante árboles de decisión utilizando método de división CHAID. Para estudiar las comparaciones entre grupos en los afectos, los relaciones con los iguales y el uso de Internet, se realizó la prueba no paramétrica Kruskal-Wallis, dado que los datos no presentaron una distribución normal (Kolmogorov-Smirnov <.05), así, como el análisis de estimación del tamaño del efecto, mediante el cálculo de la g de Hedges (.04: mínima necesaria, .25: moderada, .64: fuerte).

\section{Resultados}

Prevalencia de ciberacoso antes y durante el confinamiento

Antes del confinamiento el 16.2\% $(n=95)$ de los participantes sufrieron cibervictimización y el $6.7 \%$ acosaron a otros compañeros $(n=39)$. El $8.4 \%$ $(n=49)$ se ubicó en el rol de víctima/agresores y el $68.8 \%(n=403) \sin$ rol. Esta clasificación, según el análisis discriminante, fue acertada en un 93.3\% ( $F$ de entrada $=241.37 ; p<.05 ; F$ de salida $=178.27 ; p<.05)$, según los criterios de Gil-Flores et al. (2001). Durante el confinamiento, los porcentajes de víctimas y víctimas/agresores descendieron, pasando a ser del 8.0\% ( $n=$ 47) y del 3.9\% $(n=23)$ respectivamente, en cambio el de agresores y $\sin$ rol aumentó, subiendo al $8.7 \%(n=51)$ y al $79.4 \%(n=465)$. Este modelo de roles fue acertado en un $96.4 \%$ ( $F$ de entrada $=255.58 ; p<.05 ; F$ de salida $=236.23 ; p<.05)$, según los criterios de Gil-Flores et al. (2001). Estas 
diferencias observadas entre los perfiles antes y durante el confinamiento fueron significativas según el valor de $c^{2}(9)=348.11 ; p<.05$.

\section{Perfiles de ciberacoso antes y durante el confinamiento}

Para intentar trazar perfiles de los diferentes roles (variable criterio) implicados en el ciberacoso, se utilizó la técnica de clasificación del árbol (Figura 1). Se introdujeron las variables predictoras: estudios de Grado o de Máster, sexo, edad, orientación sexual, estado civil, trabajo, valoración de las relaciones con los iguales, así como los afectos positivos y negativos en las relaciones con los compañeros, contribuyendo al modelo final: el sexo, la edad y los afectos positivos. Así se trazó el siguiente perfil de víctima: mujer (18.7\%), con poca presencia de afectos positivos respecto a las relaciones con los iguales (24.3\%) y con una edad menor de 19 años $(15.3 \%)$.

Aunque la mayoría de los agresores fueron varones (13.5\%), un 5.1\% eran mujeres, con afectos positivos por debajo de la media (5.6\%) y mayores de 19 años (8.1\%). Las víctimas/agresores fueron en su mayoría mujeres $(8.6 \%)$, con puntuaciones iguales o inferiores a 25 en los afectos positivos $(14.7 \%)$ y eran menores de 19 años.

El 73.9\% de los participantes sin rol fueron hombres y el 67.9\% mujeres que puntuaron por encima de la media en afectos positivos en las relaciones con los iguales. El modelo clasificó de forma correcta aproximadamente, al $69.1 \%$ de los individuos en general (estimación de riesgo =.309) (Berlanga et al., 2013).

Introduciendo como variable de influencia el tiempo de confinamiento, el diagrama del árbol dio lugar a los perfiles de ciberacoso durante el confinamiento recogidos en la Figura 2. Se introdujeron las variables predictoras: estudios de Grado o de Máster, sexo, edad, orientación sexual, estado civil, trabajo, valoración de las relaciones con los iguales antes del confinamiento y durante el confinamiento y los afectos positivos y negativos en las relaciones con los compañeros. Contribuyendo al modelo final: la edad, los afectos negativos y relaciones con los iguales durante el confinamiento. Respecto a las víctimas se pudo observar que la mayoría puntuaron alto - entre 33 y 50 puntos- en afectos negativos $(41.1 \%)$. También se observó que el $6.2 \%$ puntuó entre 23 y 33 puntos y eran menores de 21 años (6.9\%). Apareciendo un tercer grupo de víctimas $(4.7 \%)$ con una puntuación menor o igual a 18 que valoraba como muy negativas las relaciones con los iguales $(8.2 \%)$.

El 21.3\% de los agresores puntuaron entre 33-50 en afectos negativos. El 9.7\% puntuó entre 23 y 33 puntos y eran mayores de 21 años (10.8\%). El 3.6\% con una puntuación igual o menor a 18 que valoraba como muy negativas las relaciones con los iguales $(4.1 \%)$. 
El $6.8 \%$ de las víctimas/agresores puntuó entre 23 y 33 puntos en los afectos negativos y eran menores de 21 años (10.8\%). Encontrando un $1.8 \%$ con una puntuación igual o menor a 18 que valoraba como negativas las relaciones con los iguales $(4.2 \%)$.

El 89.9\% de los participantes sin rol puntuaron igual o por debajo de 18 en los afectos negativos y valoraban las relaciones con los iguales como negativas $(93.0 \%)$. El 77.0\% de los estudiantes sin rol puntuaron entre 23 y 33 puntos y tenían más de 21 años (82.4\%). El modelo clasificó de forma correcta, aproximadamente, al $79.1 \%$ de los individuos en general (estimación de riesgo =.209) (Berlanga et al., 2013) (figs. 1 y 2).

\section{Figura 1}

Diagrama de árbol de los roles en el ciberacoso antes del confinamiento

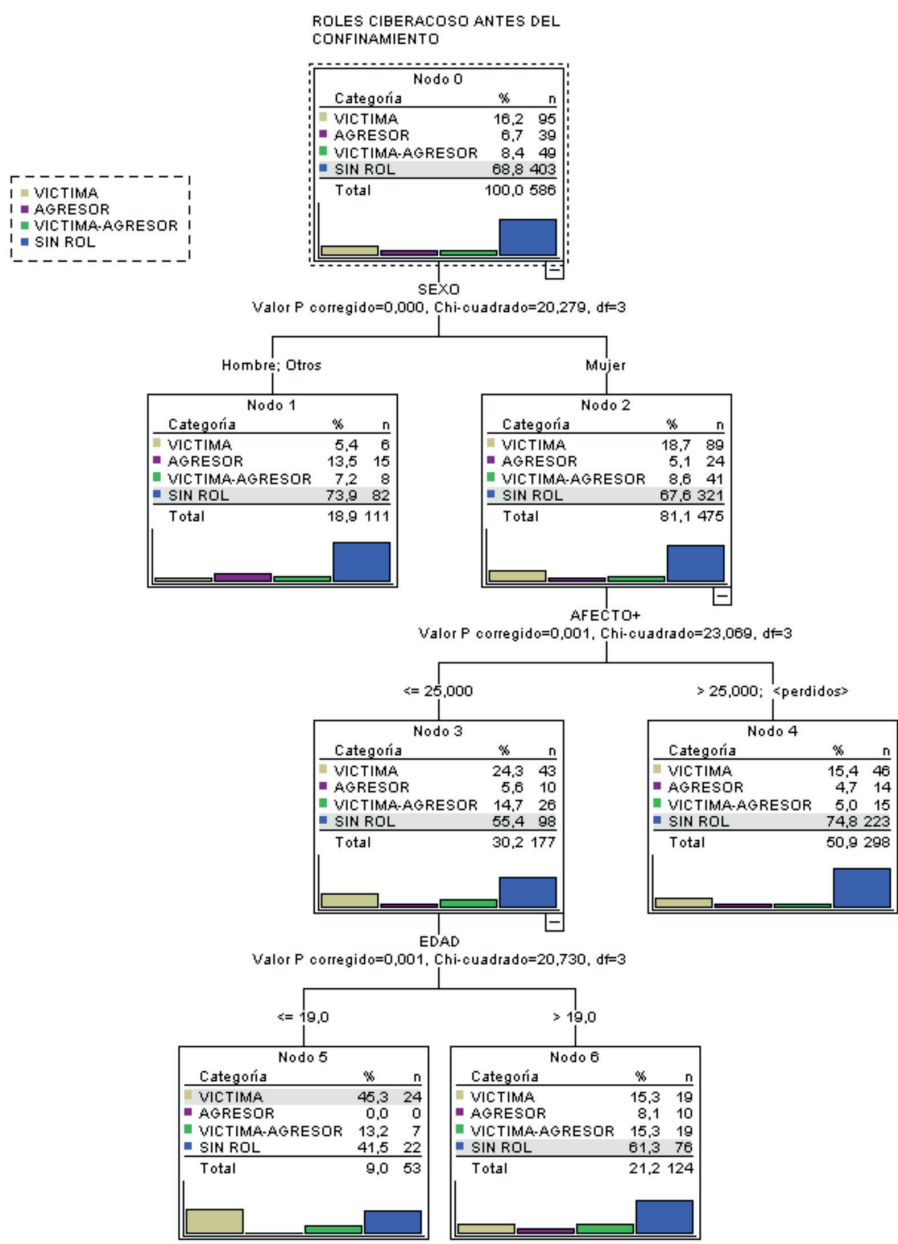




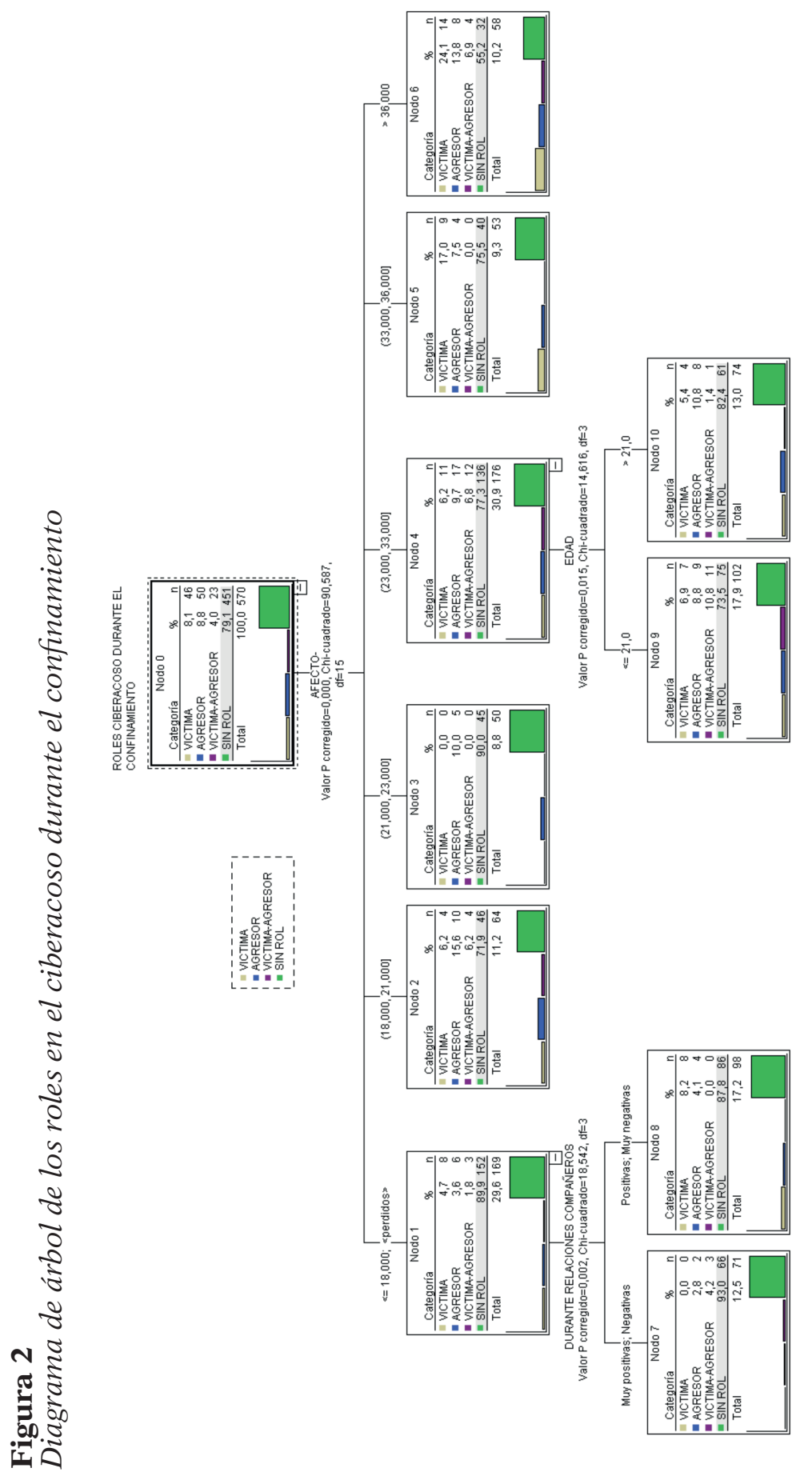




\section{Afectos experimentados antes y durante el confinamiento}

Como se ha observado en ambos árboles los afectos influyeron en los roles de ciberacoso antes y después del confinamiento, por ello se consideró importante concretar cuáles han sentido principalmente en cada rol. La prueba de Kruskal-Wallis reveló diferencias estadísticamente significativas en los afectos antes de confinamiento (Tabla 1). Las víctimas fueron las que más se sintieron angustiadas, culpables, asustadas, irritables, avergonzadas, agitadas y miedosas en comparación con los participantes sin rol. En cambio los agresores y las victimas/agresores fueron los que más agresivos se sintieron en comparación con los participantes sin rol. Además, los identificados como víctimas/agresores fueron los que menos satisfechos se sintieron comparados con los sin rol.

Tabla 1

Afectos por roles hacia las relaciones con los compañeros antes del confinamiento

\begin{tabular}{|c|c|c|c|c|c|c|c|c|c|c|c|}
\hline \multirow[t]{2}{*}{ Afectos } & \multicolumn{2}{|c|}{ Víctima } & \multicolumn{2}{|c|}{ Agresor } & \multicolumn{2}{|c|}{ V/A } & \multicolumn{2}{|c|}{ Sin rol } & \multirow[t]{2}{*}{$c^{2}$} & \multirow[t]{2}{*}{$p$} & \multirow[t]{2}{*}{$g$} \\
\hline & M & DT & $\mathbf{M}$ & DT & M & DT & M & DT & & & \\
\hline Interesado/a & 3.25 & 1.13 & 3.28 & 1.28 & 3.14 & .84 & 3.36 & 1.03 & 3.514 & .319 & .06 \\
\hline Angustiado/a & 3.26 & 1.42 & 3.26 & 1.42 & 3.24 & 1.33 & 2.87 & 1.30 & 1.390 & $.016 *$ & .23 \\
\hline Ilusionado/a & 2.43 & 1.24 & 2.44 & 1.07 & 2.27 & .99 & 2.61 & 1.19 & 5.088 & .165 & .12 \\
\hline Afectado/a & 3.19 & 1.37 & 3.10 & 1.29 & 3.27 & 1.19 & 2.93 & 1.33 & 4.892 & .180 & .11 \\
\hline Fuerte & 2.73 & 1.20 & 2.97 & 1.01 & 2.90 & 1.01 & 3.03 & 1.14 & 5.678 & .128 & .14 \\
\hline Culpable & 1.96 & 1.23 & 1.97 & 1.16 & 1.73 & .91 & 1.54 & .97 & 2.548 & $.000 *$ & .34 \\
\hline Asustado/a & 2.72 & 1.42 & 2.36 & 1.37 & 2.76 & 1.234 & 2.32 & 1.345 & 1.939 & $.012 *$ & .24 \\
\hline Agresivo/a & 1.75 & 1.05 & 2.03 & 1.31 & 2.16 & 1.12 & 1.55 & .90 & 23.020 & $.000 *$ & .38 \\
\hline Entusiasmado & 2.27 & 1.22 & 2.51 & 1.12 & 2.06 & .94 & 2.39 & 1.13 & 5.294 & .151 & .13 \\
\hline Satisfecho/a & 2.95 & 1.28 & 2.95 & 1.03 & 2.76 & 1.07 & 3.23 & 1.13 & 13.082 & $.004 *$ & .27 \\
\hline Irritable & 3.19 & 1.46 & 3.05 & 1.49 & 3.10 & 1.30 & 2.71 & 1.37 & 11.696 & $.009 *$ & .25 \\
\hline Despierto/a & 2.78 & 1.27 & 2.92 & 1.33 & 2.57 & .96 & 2.91 & 1.10 & 5.235 & .155 & .12 \\
\hline Avergonzado/a & 1.69 & .98 & 1.92 & 1.18 & 1.80 & 1.02 & 1.55 & .89 & 7.981 & $.046^{*}$ & .19 \\
\hline Inspirado/a & 2.33 & 1.24 & 2.51 & 1.12 & 2.35 & 1.13 & 2.59 & 1.12 & 5.938 & .115 & .14 \\
\hline Nervioso/a & 3.49 & 1.44 & 3.23 & 1.27 & 3.35 & 1.23 & 3.14 & 1.34 & 6.374 & .095 & .15 \\
\hline Decidido/a & 2.62 & 1.18 & 2.74 & 1.27 & 2.37 & 1.06 & 2.79 & 1.06 & 8.062 & $.045^{*}$ & .19 \\
\hline Concentrado/a & 2.33 & 1.13 & 2.54 & 1.17 & 2.18 & .99 & 2.50 & 1.12 & 5.181 & .159 & .12 \\
\hline Agitado/a & 2.97 & 1.33 & 2.64 & 1.41 & 2.84 & 1.21 & 2.53 & 1.33 & 9.781 & $.021 *$ & .22 \\
\hline Activo/a & 2.89 & 1.31 & 2.77 & 1.11 & 2.63 & .91 & 3.02 & 1.13 & 7.462 & .059 & .18 \\
\hline Miedoso/a & 2.82 & 1.47 & 2.36 & 1.41 & 2.73 & 1.38 & 2.28 & 1.32 & 14.301 & $.003 *$ & .28 \\
\hline
\end{tabular}

Nota. $M=$ Media; $D T=$ Desviación típica; V/A = Víctima/Agresor; * = significativo a.05 
La prueba de Kruskal-Wallis para los afectos durante el confinamiento mostró diferencias estadísticamente significativas (Tabla 2). Las víctimas se sintieron más afectadas, asustadas, nerviosas, irritables y agitadas que los participantes sin rol; y más activas que los agresores. Los participantes sin rol fueron los que menos angustiados se sintieron en comparación con las víctimas y los agresores; y los que menos culpables se mostraron frente a los agresores y las víctimas/agresores. Los agresores fueron los que más agresividad manifestaron comparados con los estudiantes sin rol. Las víctimas/agresores y los participantes sin rol se mostraron menos miedosos que las víctimas. Los agresores y los participantes sin rol fueron los que menos avergonzados se sintieron comparados con las víctimas.

Tabla 2

Afectos por roles hacia las relaciones con los compañeros durante el confinamiento

\begin{tabular}{|c|c|c|c|c|c|c|c|c|c|c|c|}
\hline \multirow[t]{2}{*}{ Afectos } & \multicolumn{2}{|c|}{ Víctima } & \multicolumn{2}{|c|}{ Agresor } & \multicolumn{2}{|c|}{$\begin{array}{l}\text { Víctima/ } \\
\text { Agresor }\end{array}$} & \multicolumn{2}{|c|}{ Sin rol } & \multirow[t]{2}{*}{$c^{2}$} & \multirow[t]{2}{*}{$p$} & \multirow[t]{2}{*}{$g$} \\
\hline & M & DT & M & DT & M & DT & M & DT & & & \\
\hline Interesado/a & 3.26 & .97 & 2.96 & 1.26 & 3.57 & .73 & 3.35 & 1.04 & 5.630 & .131 & .14 \\
\hline Angustiado/a & 3.60 & 1.42 & 3.39 & 1.28 & 3.26 & 1.28 & 2.88 & 1.31 & 18.944 & $.000 *$ & .34 \\
\hline Ilusionado/a & 2.55 & 1.30 & 2.18 & 1.01 & 2.74 & 1.01 & 2.57 & 1.18 & 5.899 & .117 & .14 \\
\hline Afectado/a & 3.47 & 1.41 & 3.35 & 1.23 & 3.35 & 1.23 & 2.91 & 1.32 & 12.899 & $.005 *$ & .26 \\
\hline Fuerte & 2.64 & 1.17 & 2.75 & 1.05 & 3.17 & 1.23 & 3.01 & 1.13 & 7.764 & .051 & .18 \\
\hline Culpable & 2.55 & 1.35 & 1.86 & 1.08 & 1.83 & .94 & 1.53 & .95 & 41.770 & $.000 *$ & .53 \\
\hline Asustado/a & 3.02 & 1.48 & 2.76 & 1.31 & 2.52 & 1.34 & 2.32 & 1.33 & 14.888 & $.002 *$ & .29 \\
\hline Agresivo/a & 1.91 & 1.16 & 2.04 & 1.20 & 1.87 & .82 & 1.59 & .95 & 14.148 & $.003 *$ & .38 \\
\hline Entusiasmado & 2.49 & 1.40 & 2.02 & 1.05 & 2.74 & 1.01 & 2.36 & 1.11 & 7.764 & .051 & .18 \\
\hline Satisfecho/a & 3.23 & 1.43 & 2.86 & 1.11 & 2.96 & 1.15 & 3.15 & 1.12 & 4.451 & .217 & .10 \\
\hline Irritable & 3.47 & 1.46 & 3.24 & 1.32 & 3.04 & 1.30 & 2.73 & 1.38 & 16.786 & $.001 *$ & .32 \\
\hline Despierto/a & 3.13 & 1.36 & 2.67 & 1.18 & 2.70 & 1.06 & 2.86 & 1.11 & 4.807 & .186 & .11 \\
\hline Avergonzado/a & 2.13 & 1.21 & 1.59 & .98 & 2.35 & 1.19 & 1.53 & .87 & 26.534 & $.000 *$ & .41 \\
\hline Inspirado/a & 2.70 & 1.30 & 2.29 & 1.12 & 2.57 & 1.31 & 2.53 & 1.12 & 3.166 & .367 & .03 \\
\hline Nervioso/a & 3.96 & 1.27 & 3.35 & 1.26 & 3.39 & 1.20 & 3.13 & 1.35 & 18.292 & $.000 *$ & .33 \\
\hline Decidido/a & 2.87 & 1.23 & 2.31 & 1.12 & 2.87 & 1.29 & 2.75 & 1.06 & 7.754 & .051 & .18 \\
\hline Concentrado/a & 2.55 & 1.25 & 2.18 & 1.09 & 2.48 & 1.08 & 2.46 & 1.09 & 3.322 & .345 & .05 \\
\hline Agitado/a & 3.28 & 1.36 & 2.71 & 1.28 & 2.83 & 1.40 & 2.55 & 1.32 & 12.497 & $.006 *$ & .26 \\
\hline Activo/a & 3.28 & 1.26 & 2.57 & 1.22 & 2.83 & 1.03 & 2.97 & 1.12 & 9.065 & $.028 *$ & .21 \\
\hline Miedoso/a & 3.26 & 1.38 & 2.78 & 1.40 & 2.35 & 1.30 & 2.29 & 1.34 & 24.938 & $.000 *$ & .40 \\
\hline
\end{tabular}

Nota. $M=$ Media; $D T=$ Desviación típica; * = significativo a. 05 
Relaciones con los iguales antes y durante el confinamiento y la docencia online

En cuanto a las relaciones con los iguales, fueron valoradas como positivas antes del confinamiento $(M=2.13 ; D T=.83$; Mínimo $=0$ y Máximo $=3$ ) por el $88.3 \%$ de los participantes y negativas por el $11.8 \%$. Durante el confinamiento, el $85.8 \%$ las consideró positivas $(M=2.13 ; D T=.83$, Mínimo = 0 y Máximo = 3) y el $14.3 \%$ negativas. Sin embargo, respecto al rol la valoración de las relaciones con los compañeros antes y durante el confinamiento no se mostraron determinantes.

También, se preguntó por las relaciones con los iguales durante la docencia online en el confinamiento, observando que solamente se hallaron diferencias significativas en "Mis compañeros me han apoyado" $\left(\boldsymbol{c}^{2}(3)=\right.$ 9.255; $p=.026 ; g=.21)$. Así los agresores $(M=3.44 ; D T=1.04)$ manifestaron que habían recibido un menor apoyo que el resto de los participantes (Víctimas: $M=4.13 ; D T=1.04$; Víctimas/agresores: $M=4.08 ; D T=.89$; $\operatorname{Sin}$ rol: $M=4.02 ; D T=.92)$.

\section{Usos de Internet durante el confinamiento}

Para conocer el uso de Internet durante el confinamiento en función del rol, se realizó la prueba de Kruskal-Wallis. La misma reveló que el uso que se hacía de Internet por parte de los diferentes participantes era similar, a excepción del uso "Para contactar con mis compañeros de universidad" $\left(c^{2}(3)=9.624 ; p=.022 ; g=.23\right)$ más realizado por las víctimas $(M=4.58 ; D T$ $=0.81)$ que por los agresores $(M=3.88 ; D T=1.30)$ y los participantes $\sin$ rol $(M=4.28 ; D T=1.03)$. Así como la utilización del correo electrónico $\left(\boldsymbol{c}^{2}\right.$ (3) $=10.577 ; p=.014 ; g=.24)$ que fue menor por las víctimas/agresores $(M$ $=4.21 ; D T=.85)$ en comparación con el resto de los roles: agresores $(M=$ $4.73 ; D T=.57)$, víctimas $(M=4.72 ; D T=.45)$, y participantes sin rol $(M=$ $4.64 ; D T=.63)$.

\section{DISCUSIÓN Y CONCLUSIONES}

El estudio tuvo como objetivo analizar el ciberacoso a través del teléfono móvil en estudiantes universitarios antes y después del confinamiento provocado por la crisis sanitaria de la Covid-19. Antes del confinamiento se ha constatado la presencia de ciberacoso en el contexto de la Universidad de Granada, en una tasa algo inferior a la señalada por Vergel et al. (2016). En función del rol, los resultados de implicación fueron similares a los obtenidos por Méndez et al. (2019). 
Durante el confinamiento se esperaba (H1), tal y como estudios previos (Gómez-León, 2021; Mkhize y Gopal, 2021; Yahya et al., 2020) han referido un aumento del ciberacoso. Sin embargo, se encontró un descenso de la victimización, al igual que en estudio de Gómez-León (2021), y un aumento de los agresores. Responder al por qué ocurrió esto es una tarea compleja. Algunos de los participantes que afirmaron dejar de sufrir ciberagresiones durante el confinamiento señalaron que los compañeros los apoyaron. Estudios previos (González-Muzzio, 2013; Lozano-Díaz et al., 2020) revelan que en situaciones de crisis (guerras, terremotos, etc.) donde se necesita la colaboración de todos, se refuerza el sentimiento de utilidad y de pertenencia al grupo, y aparecen conductas emergentes para hacer frente de la mejor forma posible a la nueva situación. Sin duda las vivencias del confinamiento han podido despertar en los compañeros empatía, solidaridad, actuando como factor de protección ante el ciberacoso. Gómez-León (2021) ha revelado que durante la pandemia el apoyo familiar ha contribuido al descenso del ciberacoso en adolescentes. En este sentido, un alto porcentaje de los estudiantes de la Universidad de Granada son de otras provincias españolas, por lo que el volver a casa y estar más arropados por su familiares, también ha podido ser un punto fuerte para mejorar la capacidad de resiliencia.

En cambio, la explicación del aumento de las ciberagresiones puede encontrarse justamente en la falta de apoyo que señalaron los agresores del estudio. Resultado coincidente con los estudios previos (Calvete et al., 2010; Katzer et al., 2009; Martínez-Monteagudo, 2020) que revelan que la baja aceptación en el grupo y un bajo nivel de apoyo social se relaciona con la ciberagresión. Se trazaron perfiles diferentes en función del rol desarrollado en las situaciones de ciberacoso antes y después del confinamiento, confirmándose la segunda hipótesis. Las victimas antes del confinamiento eran de sexo femenino, al igual que en los estudios de Martínez-Ramón et al. (2019), Méndez et al. (2019) y Rodríguez-Correa y Rivaudalla (2018). Experimentaban afectos positivos por debajo de la media, tal y como refieren Finkelhor et al. (2000), Beran y Li (2005), McLoughlin et al. (2013) y Rodríguez-Correa y Rivaudalla (2018). Tenían 19 años o menos, poniendo de manifiesto al igual que en estudios previos (Méndez et al., 2019) que al inicio de los estudios es cuando se evidencia mayor presencia de la victimización, y que se trasladan patrones de victimización de la Educación Secundaria a la Universidad (Kraf y Wang, 2010; Zalaquett y Chatters, 2014; Watts et al., 2017). Por su parte, los agresores antes del confinamiento fueron principalmente hombres, hallazgo coincidentes con los encontrado por Durán-Segura y Martínez-Pecino (2015), Navarro et al. (2016) y Sánchez et al. (2011).

Durante el confinamiento, no se observó una influencia del sexo al igual que en el estudio de Jain et al. (2020). La edad de las víctimas aumentó hasta los 21 años, y puntuaron alto en afectos negativos, tal y como afirman 
Ortega-Ruiz et al. (2012). Los agresores eran mayores de 21 años y también experimentaban afectos negativos - aunque en menor medida que las víctimas-. Este estado emocional sumado a la percepción de bajo apoyo social por parte de los compañeros puede hacer que se involucren más en las ciberagresiones (Romera et al., 2016). Estos perfiles relativos a la edad podrían ir en consonancia con los autores que afirman que seguirá ascendiendo (Dobarro et al., 2018), por lo tanto es necesario continuar en esta línea de estudios para clarificar el peso de la edad en el ciberacoso.

Tal y como se hipoteizó (H4) las víctimas experimentaron más afectos negativos, como señalan Ortega-Ruiz et al. (2012), Finkelhor et al. (2000) y Khine et al. (2020). Aunque se puede afirmar que los afectos negativos (asustadas, nerviosas, agitadas,...) experimentados por la víctima han sido los mismos antes y durante la pandemia. No cabe duda de que se intensificaron durante la misma (Ozamiz-Etxebarria et al., 2020), pues a la afectación por la situación de confinamiento se le suma la afectación negativa por la vivencia del ciberacoso (Jain et al., 2020).

En cuanto a la valoración de las relaciones sociales antes y durante el confinamiento no se confirmó la hipótesis 5 , ya que no se hallaron diferencias estadísticamente significativas por roles. En general, la valoración de los participantes de las relaciones con los iguales fue positiva, aunque ligeramente mejor antes del confinamiento. En cuanto a la relaciones con los iguales durante la docencia online, se observó una tendencia opuesta a lo esperado (H6), pues fueron los agresores los que manifestaron un menor apoyo social. Este hallazgo está en consonancia con los estudios que señalan su bajo apoyo social (Calvete et al., 2010; Katzer et al., 2009).

Por último, se puede hablar de un uso muy similar de Internet durante el confinamiento por parte de los diferentes roles, relacionado principalmente con el seguimiento de la docencia online, mantenerse en contacto con familiares y amigos, acceder a las redes sociales, y música y/o TV en streaming. Estos usos están en consonancia con los apuntados por el estudio de Smartme Analytics (2020). Es destacable uno de los usos diferenciales, respecto a un mayor uso por parte de las víctimas para contactar con los compañeros de universidad, lo que puede estar relacionado con una percepción más positiva del apoyo social recibido.

Entre las limitaciones del estudio pueden señalarse haber utilizado como único método de recogida de información autoinformes, ya que podrían estar influidos por la deseabilidad social, incluso tratándose de medidas válidas y fiables. Aun así, el hecho de haberlos administrado de manera online ha podido favorecer la libertad de expresión, al no sentirse observados mientras los cumplimentaban. Además, solamente se ha evaluado el ciberacoso a través 
del teléfono móvil, y no a través de otros dispositivos electrónicos, por lo que la incidencia podría ser superior. Se optó por evaluarlo a través de este medio, porque los estudiantes universitarios llevan encima constantemente el teléfono móvil (Polo et al., 2017), es la pantalla que nunca se apaga (Sádaba y Bringué, 2010), lo que permite estar expuesto y/o participar en las ciberagresiones en cualquier momento y lugar, y de forma ininterrumpida. En futuros estudios sería interesante completar esta perspectiva con la inclusión de otros dispositivos con conexión a Internet. Otra limitación está relacionada con el tipo de muestreo realizado y el tamaño y la representatividad de la muestra, por lo que se debe ser cauteloso con la generalización de los resultados. Sin embargo, dadas las circunstancias excepcionales en las que se realizó la recolección de datos, el acceso a la muestra fue más complejo.

Para futuros estudios, sería interesante incluir un instrumento que permitiera identificar el rol de los espectadores del ciberacoso, y su posicionamiento a favor de la víctima o del agresor. En el estudio realizado los participantes que fueron identificados como sin rol, según los estudios clásicos sobre el acoso, realmente tendrían un rol, el de observadores, y su papel puede ser tan decisivo para la resolución exitosa del ciberacoso, que es importante obtener más información al respecto. Pues como señala Vallés (2014), el ciberacoso continuará solamente si los espectadores lo toleran.

Como conclusión del estudio puede señalarse que la variable tiempo de confinamiento ha incidido en las situaciones de ciberacoso entre universitarios, y ha trazado perfiles diferentes de participantes en función de los afectos negativos, las relaciones con los iguales y la edad; así como que el desgaste emocional ha sido mayor durante la situación de aislamiento social por la Covid19 , pues en los roles de víctima, agresor y víctima aumentaron las emociones negativas, como angustia, afectación, nerviosismo, miedo, irritabilidad, etc.

Dado que, como se ha visto, el ciberacoso está presente en el contexto universitario, pese a la supuesta madurez de los adultos jóvenes, es necesario incidir en su formación para hacer un uso responsable, positivo y no dañino de los teléfonos móviles y demás dispositivos electrónicos con conexión a Internet y un disfrute saludable de las ciberrelaciones sociales.

Desde las universidades debe impulsarse la convivencia, y hoy más que nunca la ciberconvivencia, promoviendo campañas de sensibilización, reforzándose los valores de convivencia y los principios morales y éticos, e implementándose medidas de intervención para evitar el abandono de estudios, etc. (Méndez et al., 2019; Redondo et al., 2017). Así, como poner en marcha iniciativas basadas en la mediación entre iguales, el aprendizaje cooperativo, etc., dada la importancia del apoyo social en la prevención del ciberacoso (Bernardo et al., 2020). 


\section{REFERENCIAS BIBLIOGRÁFICAS}

Abu Bakar, H. S. (2015). The emergence themes of cyberbullying among adolescences. International Journal of Adolescence and Youth, 20(4), 393406. https://doi.org/10.1080/02673843. 2014.992027

Alsawalqa, R. O. (2021). Cyberbullying, social stigma, and self-esteem: the impact of COVID-19 on students from East and Southeast Asia at the University of Jordan. Heliyon, 7,113, e06711. https://doi.org/10.1016/j. heliyon.2021.e06711

Avilés, J. M. (2013). Análisis psicosocial del ciberbullying: Claves para una educación moral. Papeles del Psicólogo, 34(1), 65-73. https://bit.ly/36llm4H

Baldry, A. C., Farrington, D., \& Sorrentino, A. (2015). "Am I at risk of cyberbullying?” A narrative review and conceptual framework for research on risk of cyberbullying and cybervictimization: The risk and needs assessment approach. Aggression and Violent Behavior, 23, 36-51. https://doi. org/10.1016/j.avb.2015.05.014

Barkley, J. E., \& Lepp, A. (2013). Cellular telephone use is associated with greater sedentary behavior independent of leisure-time physical activity. Applied Physiology, Nutrition, and Metabolism, 38(1), 1023.

Beran, T., \& Li, Q. (2005). CyberHarassment: A study of a new method for an old behavior. Journal of Educational Computing Research, 32(3), 265-277. https://doi. org/10.21 90/8YQM-B04H-PG4DBLLH

Berlanga, V., Rubio, M. J., \& Vilá, R. (2013). Cómo aplicar árboles de decisión en SPSS. Revista d'Innovació i Recerca en Educació,
6(1), 65-79. https://doi.org/10.1344/ reire2013.6.1615

Bernardo, A. B., Tuero, E., Cervero, A., Dobarro, A., \& Galve-González, C. (2020). Acoso y ciberacoso: Variables de influencia en el abandono universitario. Comunicar, 64, 63-72. https://doi.org/10.3916/C64-2020-06

Buelga, S., \& Pons, J. (2012). Agresiones entre adolescentes a través del teléfono móvil y de internet. Psychosocial Intervention, 21(1), 91-101. https://doi. org/10.5093/in2012v21n1a2

Buelga, S., Cava, M. J., \& Musitu, G. (2012). Validación de la escala de victimización entre adolescentes a través del teléfono móvil y de internet. Pan American Journal of Public Health, 32, 36-42. https://doi.org/10.1590/ S1020-49892012000700006

Calvete, E., Orue, I., Estévez, A., Villardón, L., \& Padilla, P. (2010). Cyberbullying in adolescents: Modalities and aggressors' profile. Computers in Human Behavior, 26(5), 1128-1135. https://doi.org/10.1016/j. chb.2010.03.017

Dennehy, R., Meanney, S., Walsh, K.A., Sinnott, C., Cronin, M., \& Arensman, E. (2020). Young people's conceptualizations of the nature of cyberbullying: A systematic review and synthesis of qualitative research. Aggression and Violent Behavior, 51, 101379. https://doi.org/10.1016/j. avb.2020.101379

Dobarro, A., Carbajal, R., Ayala, I., Herrero, J., \& Bernardo, A. (2017). Ciberbullying y violencia en la universidad, ¿cómo afecta al abandono? Revista de Estudios e Investigación en Psicología y Educación, 
vol. extr.(14), 182-185. https://doi. org/10.17979/reipe.2017.0.14.2737

Dobarro, A., Tuero, E., Bernardo, A. B., Herrero F. J., \& Álvarez-García, D. (2018). Un estudio innovador sobre acoso on-line en estudiantes universitarios. Revista d'Innovació Docent Universitària, 10, 131-142.

Durán-Segura, M., \& Martínez-Pecino, R. (2015). Ciberacoso mediante teléfono móvil e Internet en las relaciones de noviazgo entre jóvenes. Comunicar, 44, 159-167. https://doi. org/10.3916/C44-2015-17

Faucher C., Jackson, M., \& Cassidy, W. (2014). Cyberbullying among university students: gendered experiences, impacts, and perspectives. Education Research International, 2014, 1-11. https://doi. org/10.1155/2014/698545

Finkelhor, D., Mitchell, K. J., \& Wolak, J. (2000). Online victimization: A report on the nation's youth. National Center for Missing \& Exploited Children. https://bit.ly/3mZw6vv

Gámez-Guadix, M., Orue, I., Smith, P. K., \& Calvete, E. (2013). Longitudinal and reciprocal relations of cyberbullying with depression, substance use, and problematic internet use among adolescents. Journal of Adolescent Health, 53(4), 446-452. https://doi. org/10.1016/j.jadohealth.2013.03.030

Garaigordobil, M., \& Oñederra, J.A. (2009). Acoso y violencia escolar en la comunidad autónoma del País Vasco. Psicothema, 21(1), 83-89. https://bit. ly/36fH5Lg

Gómez-León, M. I. (2021). Disminución de la ansiedad en las víctimas del bullying durante el confinamiento por COVID-19. RED, 65(21), 1-20. https:// doi.org/10.6018/red.439601
González-Muzzio, C. (2013). El rol del lugar y el capital social en la resiliencia comunitaria posdesastre. Aproximación mediante un estudio de caso después del terremoto del 23/F. EURES, Revista Latinoamericana de Estudios Urbanos Regionales, 39(117), 25-48. http://dx.doi.org/10.4067/S025071612013000200002

Gil-Flores, J., García-Jiménez, E., \& Rodríguez-Gómez, G. (2001). Análisis discriminante. La Muralla.

Hernández, R., Fernández, C., \& Baptista, P. (2010). Metodología de la investigación (5th Ed.). McGrawHill.

Hoff, D. L., \& Mitchell, S. N. (2009). Cyberbullying: causes, effects, and remedies. Journal of Educational Administration, 47(5), 652-665. https:// doi.org/10.1108/09578230910981107

Jain, O., Gupta, M., Satam, S., \& Panda, S. (2020). Has the COVID-19 pandemic affected the susceptibility to cyberbullying in India? Computers in Human Behavior Reports, 2, 100029. https://doi.org/10.1016/j. chbr.2020.100029

Katzer, C., Fetchenhauer, D., \& Belschak, F. (2009). Cyberbullying: Who are the victims? A comparison of victimization in Internet chatrooms and victimization in school. Journal of Media Psychology, 21(1), 25-36. https:// doi.org/10.1027/1864-1105.21.1.25

Khine A.T., Saw Y. M., Htut Z. Y., Khaing C. T., Soe H. Z., Swe K. K., Thike, Th., Htet, H., Saw, T. N., Cho, S. M., Kariya, T., Yamamoto, E., \& Hamajima, N. (2020). Assessing risk factors and impact of cyberbullying victimization among university students in Myanmar: A crosssectional study. PLoS ONE, 15(1), 1-16, 0227051. https://doi.org/10.1371/ journal.pone.0227051 
Kiriakidis, S. P., \& Kavoura, A. (2010) Cyberbullying: A review of the literature on harassment through the internet and other electronic means. Family \& Community Health, 33(2), 82-93. https://doi.org/10.1097/ FCH.0b013e3181d593e4

Kowalski, R. M., Giumetti, G. W., Schroeder, A. N., \& Reese, H. (2012). Cyberbullying among college students: Evidence from multiple domains of college life. En C. Wankel, \& L. Wankel (Eds.), Misbehavior online in higher education (pp. 293-321). Emerald.

Kraft, E. M., \& Wang, J. (2010). An exploratory study of the cyberbullying and cyberstalking experiences and factors related to victimization of students at a public liberal arts college. International Journal of Technoethics, 1(4), 74-91. https://doi.org/10.4018/ jte. 2010100106

López-Gómez, I., Hervás, G., \& Vázquez, C. (2015). Adaptación de la "escala de afecto positivo y negativo” (PANAS) en una muestra general española. Behavioral Psychology. Psicología Conductual, 23(3), 529-548. https://bit. ly/33erppM

Lozano-Díaz, A., Fernández-Prados, J. S., Figueredo Canosa, V., \& Martínez Martínez, A. M. (2020). Impactos del confinamiento por el COVID-19 entre universitarios: Satisfacción Vital, Resiliencia y Capital Social Online. International Journal of Sociology of Education, Special Issue: COVID-19 Crisis and Socioeducative Inequalities and Strategies to Overcome them, 79-104. http://doi.org/10.17583/ rise. 2020.5925

Martínez-Monteagudo, M. C., Delgado, B., García-Fernández, J. M., \& RuízEsteban, C. (2020). Cyberbullying in the university setting. Relationship with emotional problems and adaptation to the university. Frontiers in Psychology, 10, 3074. http://doi. org/10.3389/fpsyg.2019.03074

Martínez-Ramón, J. P., Méndez, I., Ruiz-Esteban, C., \& Cerezo, F. (2020). Validación y fiabilidad del Cuestionario sobre Acoso entre Estudiantes Universitarios (QAEU). Revista Fuentes, 22(1), 88-104. https:// doi.org/10.12795/revistafuentes.2020. v22.i1.08

Martínez-Ramón, J. P., Ruíz-Esteban, C., \& Méndez-Mateo, I. (2019). Víctimas universitarias de acoso: Un estudio sobre factores temporales y situacionales. European Journal of Investigation in Health, Psychology and Education, 9(3), 149-157. http://doi. org/10.30552/ejihpe.v9i3.331

Mason, T. (2005). How to teach children at-risk of educational failure: Coping with poverty, bullying, disease, crime and ethnicity. The Edwin Mellen Press.

McLoughlin, C., Meyricke, R., \& Burgess, J. (2013). Conference paper edition: Bullies in cyberspace: how rural and regional Australian youth perceive the problem of cyberbullying and its impact. https://bit.ly/3n0FHIR

Méndez, I., Ruiz-Esteban, C., Martínez, J. P., \& Cerezo, F. (2019). Ciberacoso según características sociodemográficas y académicas en estudiantes universitarios. Revista Española de Pedagogía, 77(273), 261276. https://doi.org/10.22550/REP772-2019-06

Mkhize, S., \& Gopal, N. (2021). Cyberbullying perpetration: children and youth at risk of victimization during Covid-19 lockdown. International Journal of Criminology and Sociology, 10, 525-537. https://doi. org/10.6000/1929-4409.2021.10.61 
Myers, C. A., \& Cowie, H. (2013). University students' views on bullying from the perspective of different participant roles. Pastoral Care in Education, 31(3), 251-267. https://doi. org/10.1080/02643944.2013.811696

Navarro, R., Larrañaga, E., \& Yubero, S. (2016). Gender identity, gender-typed personality traits and school bullying: Victims, bullies and bully-victims. Child Indicators Research, 9(1), 1-20. https:// doi.org/10.1007/s12187-015-9300-z

Navarro, R., Yubero, S., \& Larrañaga, E. (2015). Psychosocial risk factors for involvement in bullying behaviors: empirical comparison between cyberbullying and social bullying victims and bullies. School Mental Health, 7, 235-248. http://dx.doi. org/10.1007/s12310-015-9157-9

Ortega-Barón, J., Buelga, S., \& Cava, M.J. (2016). The influence of school climate and family climate among adolescents victims of cyberbullying. Comunicar, 46, 57-65. http://dx.doi. org/10.3916/C46-2016-06

Ortega-Ruiz, R., Del Rey, R., \& Casas, J. A. (2012). Knowing, building and living together on internet and social networks: The ConRed cyberbullying prevention program. International Journal of Conflict and Violence, 6(2), 302-312. https://doi.org/10.4119/ UNIBI/ijcv. 250

Ozamiz-Etxebarria N., Dosil-Santamaria M., Picaza-Gorrochategui M. I., \& Mondragon N. (2020). Niveles de estrés, ansiedad y depresión en la primera fase del brote del COVID-19 en una muestra recogida en el norte de España. Cadernos Saúde Pública, 36(4), 1-9. https://doi. org/10.1590/0102-311x00054020

Patchin, J. W., \& Hinduja, S. (2012). Cyberbullying: An update and synthesis of the research. En J.
W. Patchin \& S. Hinduja (Eds.), Cyberbullying prevention and response: Expert perspectives (pp. 13-35). Routledge/Taylor \& Francis Group.

Polo, M. I., Mendo, S. L., León, B., \& Felipe, E. (2017). Abuso del móvil en estudiantes universitarios y perfiles de victimización y agresión. Adicciones, 29(4), 245-255. https://doi. org/10.20882/adicciones.837

Redondo, J., Luzardo-Briceño, M., García-Lizarazo, K. L., \& Inglés, C. J. (2017). Impacto psicológico del ciberbullying en estudiantes universitarios: Un estudio exploratorio. Revista Colombiana de Ciencias Sociales, 8(2), 458-478. https://doi. org/10.21501/22161201.2061

Rodríguez-Correa, M., \& RivadullaLópez, J. C. (2018). Percepción y experiencias sobre el ciberbullying en estudiantes universitarios. Revista d'Innovació Educativa, 21,10-22. https://doi.org/10.7203/attic.21.12926

Romera, E. M., Cano, J. J., GarcíaFernández, C. M., \& Ortega-Ruiz, R. (2016). Cyberbullying: competencia social, motivación y relaciones entre iguales. Comunicar, 48, 71-79. http:// dx.doi.org/10.3916/C48-2016-07

Sádaba, C, \& Bringué, X. (2010). Niños y adolescentes españoles ante las pantallas: Rasgos configuradores de una generación interactiva. CEE Participación Educativa, 15, 86-104. https://bit.ly/36g5lNk

Sánchez, V., Ortega, R., \& Menesini, E. (2012). La competencia emocional de agresores y víctimas de bullying. Anales de Psicología, 28(1), 71-82 https://bit.ly/2TpAgm9

Ševčíková, A., \& Šmahel, D. (2009). Online harassment and cyberbullying in the Czech Republic: Comparison across age groups. Zeitschrift für 
Psychologie/Journal of Psychology, 217(4), 227-229. https://doi. org/10.1027/0044-3409.217.4.227

Smartme Analytics (2020). Estudio del impacto del coronavirus en el uso del móvil. https://bit.ly/2RKLelS

Tindell, D. R., \& Bohlander, R. (2012). The use and abuse of cell phones and text messaging in the classroom: A survey of college students. College Teaching, 60(1),1-9. https://doi.org/10 .1080/87567555.2011.604802

Vallés, A. (2014). Emociones y sentimientos en el acoso escolar. Revista digital EOS Perú, 3(1), 7-17. https://bit.ly/33dBDqz

Vergel, M., Martínez, J., \& Zafra, S. (2016). Factors associated with bullying in higher education institutions. Revista Criminalidad, 58(2), 197-208. https://bit.ly/36dQdjH

Washington, E. T. (2015). An overview of cyberbullying in higher education. Adult Learning, 26(1), 21-27. https:// doi.org/10.1177/1045159514558412

Watts, L. K, Wagner J., Velasquez B., \& Behrens P. I. (2017). Cyberbullying in higher education: a literature review. Computers in Human Behavior, 69, 268-274. https://doi.org/10.1016/j. chb.2016.12.038

Wegge, D., Vandebosch, H., Eggermont, S., Van Rossem, R., \& Walravel, M. (2016). Divergent perspectives: Exploring a multiple informant approach to cyberbullying victimization and perpetration.
European Journal on Criminal Policy and Research, 22(2), 235-251. http:// dx.doi.org/10.1007/s10610-015-9287-5

Wensley, K., \& Campbell, M. A. (2012). Heterosexual and nonheterosexual young university students' involvement in traditional and cyber forms of bullying. Cyberpsychology, Behavior, and Social Networking, 15(12), 649-654. https://doi.org/10.1089/ cyber.2012.0132

Worsley, J. D., McIntyre, J. C., \& Rhiannon, C. (2019). Cyberbullying victimisation and mental distress: testing the moderating role of attachment security, social support, and coping styles. Emotional and Behavioural Difficulties, 24(1), 20-35. https://doi.org/10.1080/13632752.2018 .1530497

Wright M. F. (2016). Cybervictims' emotional responses, attributions, and coping strategies for cyber victimization: a qualitative approach. Safer Communities, 15(3), 160-169. https://doi.org/10.1108/SC-03-20160006

Yahya, A. S., Khawaja, S., \& Chukwuma, J. (2020). COVID-19 and a potential rise in bullying behaviors. The Primary Care Companion for CNS Disorders, 22(3), 20com02642. https://doi. org/10.4088/PCC.20com02642

Zalaquett, C. P., \& Chatters, S. J. (2014). Cyberbullying in college: frequency, characteristics, and practical implications. Sage Open, 4, 1-8. https:// doi.org/10.1177/2158244014526721 


\section{PERFIL ACADÉMICO Y PROFESIONAL DE LOS AUTORES}

María Jesús Caurcel Cara. ORCID: https://orcid.org/0000-0002-2169-9100

Profesora Contratada Doctora, Dpto. Psicología Evolutiva y de la Educación de la Universidad de Granada (España). Líneas de investigación: bullying, ciberbullying y sexting; atención a la diversidad; educación inclusiva; necesidades educativas especiales; diversidad familiar; formación del profesorado; trastornos del neurodesarrollo; problemas de aprendizaje; trastorno por déficit de atención e hiperactividad (TDAH), TIC, entre otras. E-mail: caurcel@ ugr.es

Emilio Crisol-Moya. ORCID: https://orcid.org/0000-0002-3091-6699

Profesor Contratado Doctor, Dpto. de Didáctica y Organización Escolar de la Universidad de Granada (España). Líneas de investigación: atención a la diversidad; educación inclusiva; necesidades educativas especiales; liderazgo inclusivo; formación del profesorado; educación superior; metodologías activas; TIC; tecnología educativa; metodologías activas; diversidad familiar, entre otras. E-mail: ecrisol@ugr.es

Fecha Recepción del Artículo: 30. Marzo. 2021

Fecha Modificación del Artículo: 28. Mayo. 2021

Fecha Aceptación del Artículo: 03. Junio. 2021

Fecha Revisión para Publicación: 13. Septiembre. 2021 